\title{
Distribution peculiarities of stray fields and magnetization near magnet singularities
}

\author{
V.N. Samofalov ${ }^{1}$, D.P.Belozorov ${ }^{2}$, A.G. Ravlik ${ }^{1}$, A.S. Aseev \\ ${ }^{1}$ National Technical University "Kharkiv Polytechnic Institute", \\ ${ }^{2}$ Kirpichov Str., 61002, Kharkiv, Ukraine \\ ${ }^{2}$ National Scientific Center "Kharkiv Physical and Technical Institute", \\ 1 Akademicheskaya Str. 61108, Kharkiv, Ukraine
}

\section{Received March 29, 2017}

\begin{abstract}
Distributions of both magnetization and stray fields near singularities of a permanent magnet with high uniaxial anisotropy have been studied. On the basis of calculations it is shown that in magnets with high magnetic anisotropy, strong stray fields $H>4 \pi M s$ occurring near the edge of a magnet do not practically result in deviation of magnetization from easy axis if the quality factor of the magnetic material $g=K /\left(2 \pi M S^{2}\right)$ is $g>10$. In such magnet systems, the distribution of magnetization is close to homogeneous, and it is possible to use the method of "magnetic charges" for calculations of stray fields. It is shown that the stray field near an edge of a magnet takes finite values, and the presence of a singularity at the dependence of the tangent component of the stray field $H_{\tau} \approx$ $M s \cdot \ln (a / r)$ at $r \rightarrow 0$ is related to macroscopic characteristics generally accepted in magnetism, namely the surface density of "magnetic charges" $\sigma$.

Keywords: permanent magnet, easy axis of magnetization, stray fields, high anisotropy, "magnetic charges", galvanomagnetic sensor, magnetic dipole, bismuth film.

Изучено распределение намагниченности и полей рассеяния вблизи сингулярных точек магнита с большой одноосной анизотропией. На основании проведенных расчётов показано, что в магнитах с большой магнитной анизотропией возникающие вблизи края магнита сильные поля рассеяния $H>4 \pi M s$, практически не приводят к отклонению намагниченности от лёгкой оси, если фактор качества вещества магнита $g=K /\left(2 \pi M S^{2}\right)$ больше $g>10$. В таких системах магнитов распределение намагниченности близко к однородному и при расчёте полей рассеяния можно использовать метод "магнитных зарядов". Показано, что поле рассеяния вблизи края магнита имеет конечные значения, а наличие сингулярности на зависимости касательной компоненты поля рассеяния $H_{\tau} \approx M s \cdot \ln (a / r)$ при $r \rightarrow 0$ связано с принятой в магнетизме макроскопической характеристикой $\sigma$ - поверхностная плотность "магнитных зарядов".
\end{abstract}

Особливості розподілу полів розсіювання та намагніченості поблизу сингулярних точок магніту. В.М. Салофалов, Д.П. Бєлозоров, А.Г. Равлік, А.С. Асєєв.

Вивчено розподіл намагніченості і полів розсіювання поблизу сингулярних точок магніту 3 великою одноосною анізотропією. На основі проведених розрахунків показано, що у магнітах з великою могнітною анізотропією сильні поля розсіювання $H>4 \pi M s$, що виникають поблизу краю магніту, практично не приводять до відхилення намагніченості від легкої осі, якщо фактор якості речовини магніту $g=K /\left(2 \pi M S^{2}\right)$ більш $g>10$. У таких системах магнітів розподіл намагніченості близький до однорідного і при розрахунку полів розсіювання можна використовувати метод "магнітних зарядів". Показано, що поле розсіювання поблизу краю магніту має кінцеві значення, а наявність сингулярності на залежності дотичної компоненти поля розсіювання $H_{\tau} \approx M s \cdot \ln (a / r)$ при $r \rightarrow 0$ пов'язана з прийнятою у магнетизмі макроскопічною характеристикою $\sigma$ - поверхнева щільність "магнітних зарядів". 


\section{Introduction}

In previous works [1-8], the possibility for existence of strong magnetic fields in magnet systems with giant magnetic anisotropy was grounded. Existence of such fields was established relatively recently using various experimental methods [9-12]. It should be noted that in [7] authors call magnetic field strong when its strength is higher than saturation induction of magnetic material $H>4 \pi M s$. Earlier, the possibility of existence of such fields was not been foreseen, therefore these were not been searched for. Discovery of strong fields and conditions of their existence extends possibilities for application of various systems of permanent magnets. So, for example, strong stray fields induced by this kind of magnets are also notable for high gradients $d H / d r$, and the product $H(d H / d r)$ is comparable with the value achieved in superconducting magnets with dysprosium cores at helium temperatures. The parameter $H(d H / d r)$ is very important for biological investigations because it characterizes the degree of force action of magnetic fields on such systems [13]. A possibility to obtain large localized fields in very narrow space allows us to use similar film systems in the technique of magnetic recording of information onto high-coercive carriers [14,15]. Strong magnetic fields in permanent magnets are caused by presence of giant magnetic anisotropy in them. As material for such magnets various compounds based on rare-earth elements and also Co-Pt, Fe-Pt, etc. alloys can be used. Large magnetic fields created by these magnets may be applied as an instrument to effect on various physical and biological objects.

One of the distinctive features of strong stray fields is the existence of singular points and lines on the calculated dependence of field strength $H$ on the distance $\mathrm{r}$ between the singular point of the magnet and the observation point where the field strength tends to infinity when decreasing $r$ to zero. High values of strength parameter $H(d H / d r)$ are achieved exactly near singular points and lines.

As further we'll be discussing the questions concerning singularity, we present the results of calculations of stray fields occurring near a single homogeneously magnetized magnet in the shape of a parallelepiped (Fig.1) [1]. Note that appearing of strong stray fields follows from solutions of other magneto-static problems as well $[2,4,5,7]$. Under calculations of the stray fields,

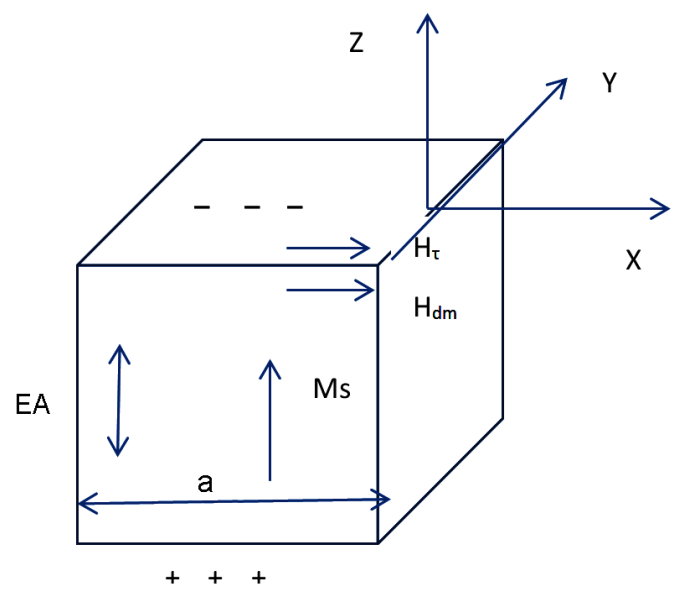

Fig. 1. Permanent magnet as parallelepiped (EA - easy axis of magnetization; $a$ - characteristic size of the magnet).

authors of [1] applied the method of "magnetic charges" with density $\sigma=\Delta M$ ( $M$ is magnetization); which occurrence follows from the continuity condition of the induction component normal to surface. Under homogeneous magnetization of a magnet (Fig.1), charges with density $\sigma= \pm M s$ ( $\sigma$ is density of magnetic poles) occur at parallelepiped faces parallel to XOY plane. If the size of the magnet along $O Y$ axis is assumed to be large and its bottom face is considered to be at a massive soft magnetic core or at infinity, the calculated dependence of the stray field tangential component $H_{\tau}$ on the distance from the magnet edge $r=\left(x^{2}+z^{2}\right)^{0.5}<<a$ will be of the form [7]:

$$
H_{\tau}=2 M s \cdot \ln (a / x)=2 M s \cdot \ln (a / r),
$$

where $a$ is a characteristic size of the magnet.

It is seen from (1) that at $r \rightarrow 0$, the stray field tangential component tends to become infinite. All the points positioned at some edges of the magnet are singular. Dependence (1) was obtained under the assumption of homogeneous magnetization of the magnet, i.e. large demagnetization fields $H_{\mathrm{dm}}$ occurred did not practically cause any deviation of magnetization from easy axis (EA). To obtain answer on the question about magnetization distribution near the magnet edge, the authors [1] have solved a variational problem. Calculations showed absence of any deviation of magnetization from EA even near the edge of the magnet. This unexpected result was explained that $H_{\tau}$ increase near the magnet edge is compensated by enlarging the ex- 
change field due to magnetization deviation from EA near singular points. As a consequence, the distribution of magnetization should remain homogeneous even at the magnet edge.

But it follows from the continuity condition for the stray field tangential component $H_{\tau}$ that the demagnetization field $H_{\mathrm{dm}}$ in the subsurface layer of the magnet (at $z \leq 0$ and small $r$ ) is also characterized by the relation (1), i.e. $H_{\tau}=H_{\mathrm{dm}}=H_{x}$. Such large demagnetization field in the magnet subsurface layer is directed perpendicular to EA and may lead to deviation (twisting) of the magnetization vector from EA in the magnet points close to its edges (axis OY). Evidently, real distribution of magnetization in materials with large but finite uniaxial anisotropy field should not be strictly homogeneous. Appearance of the magnetization inhomogeneity will result in decreasing the stray field strength near the magnet edges. Therefore, data on magnetization distribution in the vicinity of magnet singular points are of principal importance. The range of issues related to singularity of $H_{\tau}$ dependence requires detailed discussion. As the stray field may not be infinitely large for physical reasons, it is important to know what limited values it may have at the most corner point.

Stray fields can be calculated, if the magnetization direction near these singular points is known. The data obtained in [1] on the homogeneous character of magnetization distribution near singular points of magnets with high anisotropy raise doubts. Therefore, such calculation is necessary because it will allow determining the degree of the magnetization deviation from the homogeneous distribution. The following problems were put in the work:

To find the distribution of magnetization in the magnet near a singular point and determine the limiting angle $\theta_{\max }$ of magnetization deviation from EA in this point.

To find limiting values of stray field near the singular points.

\section{Peculiarities of stray fields near singular points}

As it is seen from (1) near edges of a magnet in the shape of a parallelepiped, both demagnetization and stray fields take large values $H>4 \pi M s$. In this connection, the question raises about the field strength near a corner point at distances equal to several interatomic ones. It is extraordinary

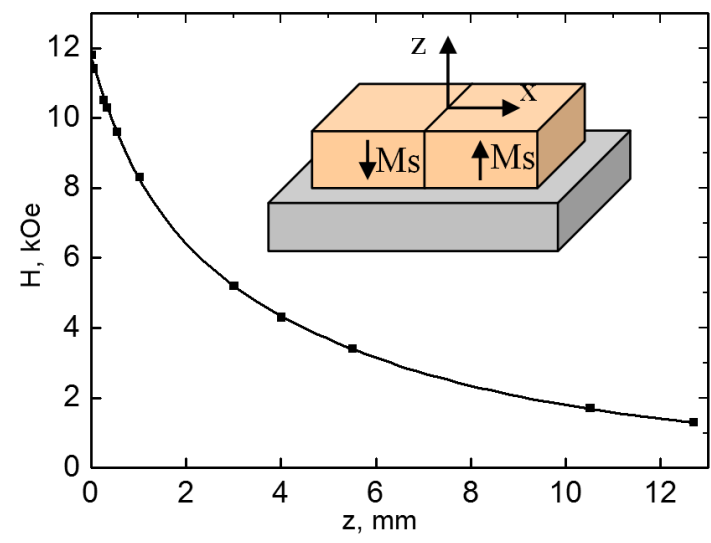

Fig. 2. Field strength $H_{X}$ dependence on distance from magnets in $z$ direction measured with $\mathrm{Bi}$ film sensor. The inset shows the scheme of used magnets.

difficult to measure the field strength at the distance from an edge in several crystalline lattice parameters. In previous works [7-10], measurements of these fields were averaged because of finite sizes of applied sensors in the form of narrow stripes with $d_{\text {min }}=14 \mu \mathrm{m}$ and $100 \mu \mathrm{m}$.

In the present work, measurements of stray fields near magnet singular points were carried out using Bi film magneto-resistive sensors. The measurements were performed for a system of two Nd-Fe-B magnets connected by a soft magnetic core. The field $H_{x}$ over the double magnet system is twice higher than over a single one; that provided higher precision of measurements. As sensors, thin $\mathrm{Bi}$ films in the form of narrow strips with width $150 \mu \mathrm{m}$ and length $3 \mathrm{~mm}$ were used. Measured values of field strength $\mathrm{Hx}$ at different distances from the magnet are given in Fig.2. These data provides an additional verification for existence of strong stray fields.

It is necessary to note that a homogeneously magnetized magnet is a metastable system because a multi-domain state is more energetically favorable. However, transition into the equilibrium multi-domain state is connected with overcoming the potential barrier $\Delta \Phi \sim H_{c}$ caused by large coercive force $H_{c}$ of the magnet material. In the modern high-energetic magnets, the coercive force under magnetization along EA is caused by the field of the inverse magnetization nuclei and their growth in the inverse field. This achieves values $H_{c}=10-80 \mathrm{kOe}$ [16]. The homogeneously magnetized state should be considered as a local minimum. Magnetization of the magnet by the field $\mathrm{H}_{\mathrm{dm}}$ directed perpendicular to EA occurs reversibly and 
without hysteresis. Therefore for such quasi-equilibrium system, directions of magnetic moments $\mu$ can be found from the condition of free energy minimum.

Under determination of directions for magnetization vectors near singular points of the magnet in the shape of a parallelepiped we considered it as a cubic lattice in which sites atoms with magnetic moments $\mu$ are positioned (Fig.3). The choice of the magnet shape was related with that the largest stray fields are achieved near an edge of the charged surface [12].

It was assumed the size of the magnet along OY axis was large; thus the position of magnetization vector at each site was characterized only by the angle $\varphi_{i, k}(x, z)$. Easy magnetization axis is parallel to $\mathrm{OZ}$ one. In the system shown in Fig.3, the deviation from EA takes place through action of the tangential component of demagnetization field $H_{\mathrm{dm}}$. The $H_{z}$ component of the demagnetization field is close to zero at the edge of the magnet $(x=0)$; in the center of the magnet $H_{z}<2 \pi M s$ is usually less than coercive force and therefore it does not change the magnetic state. The component $H_{y}=0$ due to large length of the magnet along OY. As a result of $H_{\mathrm{dm}}$ action, the magnetization near the edge of the magnet (OY axis) deviates from EA (twists). Anisotropy field $H_{k}$ and exchange field $H_{a}$ counteract the magnetization twisting; and as a result of three different field types interaction, some equilibrium state of magnetic moments $\mu$ occurs. As we are preferentially interested in the magnetization distribution near the edge of the parallelepiped, the coordinate origin is chosen at the magnet edge in the corner point of Fig.3. The size of the area where the magnetization twisting takes place was determined arbitrary by selecting the numbers $N$ and $M$ of magnetic dipoles in the lattice along $X$ and $Z$ axes respectively. Under calculations we have taken $N=M=100,1000$, and 10000 . Vector $\mu_{0}$ at the left lower corner of the lattice $(i=k$ $=0$ ) was assumed to be directed parallel to EA, i.e. $\varphi=0$. The expression for free energy $W$ of the lattice under consideration includes anisotropy energy $W_{a}=\Sigma K_{d} \sin \varphi_{i}{ }^{2}$, exchange energy $W_{E}=A_{d} \Sigma\left[\varphi_{i, k}-\varphi_{i, k-1}\right]^{2}$, and magnetic field energy $W_{H}=\Sigma-\left(\mathbf{H}_{\mathbf{i}}, \mu_{\mathbf{i}}\right)$, where $K_{d}$ and $A_{d}$ are respectively anisotropy and exchange energies per atom. Angles $\varphi_{i, k}$, and the angle $\theta_{\max }$ corresponding to the corner point $(x=0, z=0)$ were the calculated parameters. As in all rest lattice

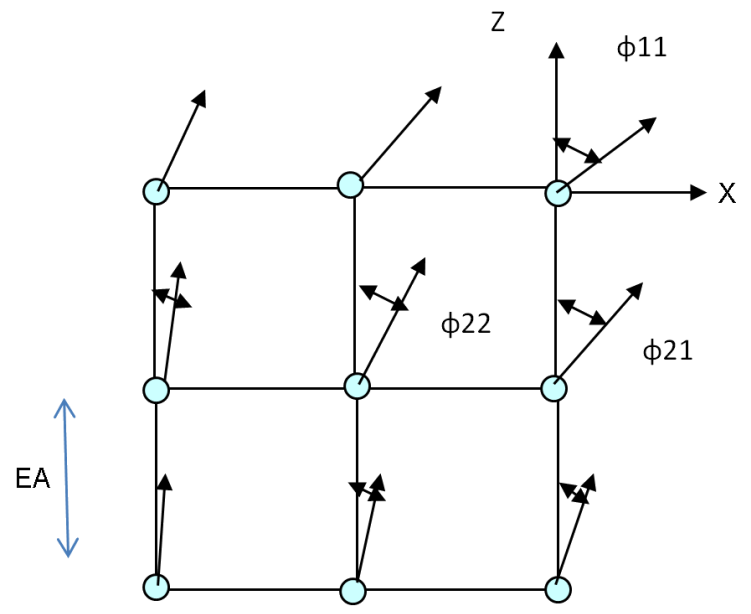

Fig. 3. The scheme of $2 \mathrm{D}$ cubic lattice in which sites atoms with magnetic moment are arranged.

sites the angle $\varphi_{i, k}<\theta_{\max }$; by the value of this angle one can evaluate the degree of the magnetization deviation from the homogeneous distribution. If $\theta_{\max }$ angle is small, the contribution of bulk charges occurring in areas with twisted magnetization ( $\operatorname{div} M \neq 0$ ) into stray fields can be ignored and only surface charges could be taken into account under calculations. In this case, the problem connected with stray field calculations becomes much simpler. At high values of the uniaxial anisotropy constant $K$ and the exchange constant $A$, the angle of magnetization deviation from EA will be small, therefore $\sin \varphi=\varphi$ may be taken.

In this extreme-value problem for $W\left(\varphi_{i, k}\right)$ function, the number of unknown parameters $S$ are equal to the number of atoms within the twisted layer, i.e. $S=N M=N^{2}$. It is difficult to find the extreme value of this function; therefore the assumption was taken that under increasing the $i, k$ coordinates, $\mu_{i}$ vector rotates by the same angle $\Delta \varphi$. Then in lattice sites with $i, k$ coordinates the angle $\varphi_{i, k}=\Delta \varphi(i+k)$, and the expression for free energy becomes

$$
\begin{aligned}
W=K_{d} \sum_{1}^{N} \sum_{1}^{M}[(i+k) \Delta \varphi]^{2}+A_{d} \cdot N \cdot M \cdot \Delta \varphi^{2}- \\
\\
-\sum_{1}^{N} \sum_{1}^{M}\left(\boldsymbol{H}_{i, k} \mu_{i k}\right)
\end{aligned}
$$

The most difficult part of the computation is precise determination of magnetic field energy $W_{H}$ because this energy depends in a complicated manner on the mag- 
Table 1. Calculated values of limit angle $\theta_{\max }$ between the magnetization vector and easy axis in a magnet shaped as parallelepiped under various values of anisotropy and exchange constants.

\begin{tabular}{||c|c|c|c|c|c|c||}
\hline № & $N=M$ & $K, \mathrm{erg} / \mathrm{at}$ & $A, \mathrm{erg} / \mathrm{at}$ & $H$, Oe & $x=\Delta \varphi, \mathrm{rad}$ & $\theta_{\max }=(N+M) \cdot \Delta \varphi, \mathrm{deg}$ \\
\hline 1 & 100 & $10^{-14}$ & $10^{-13}$ & 40000 & $3.40 \cdot 10^{-4}$ & $6.80 \cdot 10^{-2}=3.9^{\circ}$ \\
\hline 2 & 1000 & $10^{-14}$ & $10^{-13}$ & 80000 & $3.42 \cdot 10^{-5}$ & $6.84 \cdot 10^{-2}=4^{\circ}$ \\
\hline 3 & 10000 & $10^{-14}$ & $10^{-13}$ & 80000 & $3.43 \cdot 10^{-6}$ & $6.86 \cdot 10^{-2}=4.1^{\circ}$ \\
\hline 4 & 100 & $10^{-15}$ & $10^{-13}$ & 40000 & $1.69 \cdot 10^{-3}$ & $3.38 \cdot 10^{-1}=18.8^{\circ}$ \\
\hline 5 & 1000 & $10^{-15}$ & $10^{-13}$ & 40000 & $1.71 \cdot 10^{-4}$ & $3.42 \cdot 10^{-1}=19.6^{\circ}$ \\
\hline 6 & 10000 & $10^{-15}$ & $10^{-13}$ & 40000 & $1.72 \cdot 10^{-5}$ & $3.43 \cdot 10^{-1}=19.7^{\circ}$ \\
\hline 7 & 10000 & $10^{-15}$ & $10^{-13}$ & 80000 & $3.43 \cdot 10^{-5}$ & $6.86 \cdot 10^{-1}=39.5^{\circ}$ \\
\hline 8 & 10000 & $10^{-15}$ & $10^{-13}$ & 60000 & $2.57 \cdot 10^{-5}$ & $5.14 \cdot 10^{-1}=29.5^{\circ}$ \\
\hline 9 & $10^{6}$ & $10^{-15}$ & $10^{-13}$ & 60000 & $2.57 \cdot 10^{-7}$ & $5.14 \cdot 10^{-1}=29.5^{\circ}$ \\
\hline 10 & 100 & $10^{-15}$ & $10^{-14}$ & 40000 & $1.70 \cdot 10^{-3}$ & $3.42 \cdot 10^{-1}=19.5^{\circ}$ \\
\hline 11 & 10000 & $10^{-15}$ & $10^{-14}$ & 40000 & $1.72 \cdot 10^{-5}$ & $3.43 \cdot 10^{-1}=19.7^{\circ}$ \\
\hline
\end{tabular}

Table 2. Different values of the angle $\theta_{\max }$ calculated under various magnitudes of the field $H_{X}$ and the uniaxial anisotropy field $H_{K}$.

\begin{tabular}{|c|c|c|c|c|c|c|c|c|c|c|c||}
\hline$H_{K}, \mathrm{kOe}$ & 20 & 100 & 140 & 160 & 180 & 200 & 240 & 280 & 320 & 360 & 400 \\
\hline $\begin{array}{c}\theta_{\text {max }}, \mathrm{deg} \\
\left(H_{X}=80 \mathrm{kOe}\right)\end{array}$ & $>45$ & 22 & 16 & 14 & 12 & 11.5 & 9.5 & 8.2 & 7.2 & 6.4 & 5.8 \\
\hline $\begin{array}{c}\theta_{\max }, \mathrm{deg} \\
\left(H_{X}^{=} 50 \mathrm{kOe}\right)\end{array}$ & $>45$ & 13.7 & 10.1 & 8.9 & 7.9 & 7.18 & 6.01 & 5.1 & 4.5 & 4.0 & 3.6 \\
\hline $\begin{array}{c}\theta_{\max }, \mathrm{deg} \\
\left(H_{X}=35 \mathrm{kOe}\right)\end{array}$ & $>45$ & 9.6 & 7.1 & 6.26 & 5.57 & 5 & 4.2 & 3.6 & 3.17 & 2.82 & 2.54 \\
\hline \hline
\end{tabular}

netization direction in the whole magnet. To simplify the calculations, we assumed the stray field component $H_{\mathrm{dm}}$ within the lattice to be constant and deliberately overstated, for example, $H_{\mathrm{dm}}=H_{i k}=60 \mathrm{kOe}$. Under calculations, we took the following values of material constants: $K_{d}$ is anisotropy constant per atom $\left(K_{d}=10^{-14}-10^{-15}\right.$ erg/at); $A_{d}$ is the exchange constant per atom $\left(A_{d}=10^{-13}-10^{-14} \mathrm{erg} / \mathrm{at}\right) ; \mu$ is atomic magnetic moment $\left(\mu=10^{-20} \mathrm{erg} / \mathrm{Oe}\right)$. For selection of these constants we used macroscopic values of corresponding constants for materials.

Results of $\theta_{\max }$ calculations carried out for various values of uniaxial anisotropy fields $H_{K}$, and field values $H=4 \cdot 10^{4} \mathrm{Oe}$, $H=6 \cdot 104 \mathrm{Oe}$, and $H=8 \cdot 10^{4} \mathrm{Oe}$ are given in Table 1 and Table 2. The taken values of field strengths approximately correspond to values of the maximum demagnetization field created by a single magnet, a system of two magnets [7] and some complex system of Halbach cylinder type [5], respectively.
It is seen from Tables 1 and 2 that in magnets with giant magnetic anisotropy, the deviation of magnetization vector from EA is not zero as it follows from [1], and the angle $\theta_{\max }$ is several degrees. So, at $H_{K}>200 \mathrm{kOe}\left(K>1 \cdot 10^{8} \mathrm{erg} / \mathrm{cm} 3\right)$, maximum angle of $\mu$ vector deviation from EA does not exceed $\theta_{\max }<10^{\circ}$. If the angle $\theta_{\max }=10^{\circ}$, density of charges near a singular point is $\sigma \approx 0.985 \mathrm{MS}$. That is less than $2 \%$ lower than pole density limiting value which is achieved under homogeneous magnetization $\left(\sigma=M_{S}\right)$. At that, the density of bulk charges is small and is in the range $\sigma_{\mathrm{bl}}$ $\sim \Delta \varphi \cdot M s \sim 10^{-4}-10^{-6} M s$. It should be noted that the actual value of $\theta_{\max }$ will be a bit lower because the assumed field strength values $H_{X}=35, H_{X}=50$ and $H_{X}=80 \mathrm{kOe}$ for this single-magnet system are overstated while the exchange constant value $A_{d}=10^{-14} \mathrm{erg} / \mathrm{at}$ being understated.

Note, that $\theta_{\mathrm{s}}$ values calculated by StonerWohlfart formula $\sin \theta_{s}=H / H_{\kappa}$, [17] obtained 
under assumption of homogeneous rotation of the magnetization vector are $\theta_{s}=5.7$ and 11.4 in the fields $H=40 \mathrm{kOe}$ and $80 \mathrm{kOe}$ respectively while $H_{\kappa}=400 \mathrm{kOe}$. As it was expected, $\theta_{s}>\theta_{\max }$ due to absence of exchange interaction.

From the data given in Tables 1 and 2 it is seen that the angle $\theta_{\max }$ is small, therefore, the density of surface magnetic poles $\sigma=\Delta M \sim M s$ remains practically constant over the face surface; consequently, $H_{\tau}(x) \approx$ $\Delta 2 M \ln (a / x)=2 \sigma \ln (a / x)$ dependence will characterize the field in the vicinity of the singular point. At that, the singularity remains under any finite poles density and is related with a macroscopic parameter - surface charge density $\sigma$, i.e. with presence of charges in infinitely thin layer. In this fact, the limitation of the macroscopic approach becomes apparent under calculation of stray fields at magnet singular points. Notice, that such singularity of electricity field strength is characteristic for a point electric charge as it follows from Coulomb law. As strong stray fields represent some edge effect, one can consider that in a homogeneously polarized parallelepiped-shape dielectric, the dependence of electrical field strength on distance $r$ will be similar to (1).

At comparable $M_{S}$ values, the limiting field value should be higher in the $\mathrm{SmCO}_{5}$ magnet system in comparison with $\mathrm{Nd}-\mathrm{Fe}-\mathrm{B}$ one. In these, uniaxial anisotropy fields, $H_{\kappa}$, are 400 and $80 \mathrm{kOe}$, respectively. From data of Tables 1 and 2 it is seen that in magnets with $H_{K}<80 \mathrm{kOe}$ and magnetization values $M_{S} \sim 1000$ gauss, $\theta_{\max }$ angle may be large. In this case, magnetization distribution can not be considered homogeneous, and in order to determine the limiting field one should make more precise calculation of stray field strength taking into account bulk charges.

Thus, the fulfilled evaluation calculations of stray fields near singular points of magnets and its appropriate analysis allow us to conclude that large demagnetization fields in these points does not practically results in the magnetization vector deviation from EA. Analysis of data from Tables 1 and 2 indicates the magnetization distribution in high-energetic magnets to be almost homogeneous provided that the quality factor $g$ of magnetic material $(g=$ $\left.K /\left(2 \pi M_{S}^{2}\right)\right)$ is higher than 10 . In this case, stray fields will be related to surface magnetic charges which density can be found for a specified surface. These facts substan- tially simplify calculations of fields, and in some cases, allow to obtain analytical expressions for stray field strength.

One may be confronted with certain difficulties when calculating a stray field strength component $H_{x}$ near an edge of a magnet at the distance equal to a dipole lattice period. With the distance $R$ equals to several lattice periods and higher, the field $H_{x}$ can be found from the expression (3) [18] or (1):

$$
\boldsymbol{H}_{\boldsymbol{d}}=\sum_{i} \sum_{k} \sum_{l}\left(\frac{3(\mu \boldsymbol{R}) \boldsymbol{R}}{R^{5}}-\frac{\mu}{R^{3}}\right)
$$

where $R$ is the distance between a dipole with magnetic moment $\mu$ and an observation point; $i, k, l$ are dipole coordinates.

The stray field at the corner point can be presented as a sum of fields - one created by a magnet dipole in the lattice corner $H_{1}$ and another is the field of rest of dipoles of the magnet $H_{d}$. Because of uncertainty of $R$ values, the equation (3) is can not be used for calculation of $\mathrm{H}_{1}$ field strength at the corner point. The reason is that electron spin magnetic moment is not localized at a certain point of an atom, and its position is characterized by some state function. However, the fact that the electron spin magnetic moment $\mu$ is distributed with density $\rho(x, y, z)$, over $V$ area gives the opportunity to conclude that the stray field in the corner point possesses finite values. Indeed, the integral $H \sim \int \mu \cdot \rho(x, y, z) d v / R^{3}$ converges, consequently, there is no singularity in any point of a magnet. Calculation of this integral is an independent problem. Thus, in the geometrical corner of the magnet, the field $H_{x}$ is finite but its exact value is unknown.

\section{Conclusion}

In order to summarize the results of our work it is necessary to note that according to (1), the most value of stray field strength for permanent magnets with size, for example, $a=10 \mathrm{~cm}$ and magnetization $M S=1000$ gauss can not exceed $H_{X}=$ $H_{\max } \approx 30 \mathrm{kOe}$ even at the distance $r \approx 10^{-7} \mathrm{~cm}$. For the system of two such magnets with soft magnetic core, the limit field will be twice higher. In Halbach cylinder $[5,6]$ and in other complex systems of many magnets [8] with giant magnetic anisotropy ( $\mathrm{Sm}-\mathrm{Co})$, the limiting field may achieve $H_{\max } \approx 100 \mathrm{kOe}$. 


\section{References}

1. A.Thiaville, D.Tomas, J. Miltat Phys. stat. sol. (a), 170, 125 (1998)

2. W.Rave, K. Ramstock, A.Hubert, J. Magn. Magn. Mater., 183, 329 (1998)

3. A.P. Malozemoff, J.C. Slonczewski, Academic Press, New York (1979).

4. R.J. Joseph, E. Schlomann, J. Appl. Phys,. 36, 1579 (1965).

5. K. Halbach, Nucl. Instrum. Meth. Phys. Res., 169,1 (1980).

6. K. Halbach J. Appl. Phys. 57, 3605 (1985)

7. V.N. Samofalov, A.G. Ravlik, D.P Belozorov., B.A. Avramenko J. Magn. Magn. Mater., 281, 326 (2004)

8. V.N. Samofalov, D.P. Belozorov, A.G. Ravlik, Phys. Metals and Metallography, 102, 527 (2006).

9. F. Bloch, O. Cugat Eur. Phys. J.-Appl. Phys. 5,85 (1999)

10. S.I Tarapov., V.N. Samofalov, A.G. Ravlik, D.P. Belozorov, Int. J. Infrar. Millim. Waves, 24, 1082 (2003)
11. D. Belozorov, V. Derkach, G. Ermak, M. Nakhimovich, A. Ravlik, V. Samofalov, S. Tarapov, A. Zamkovoy. Int. J. Infrar. Millim. Waves, 27, 107 (2006)

12. V.N. Samofalov, D.P. Belozorov, A.G. Ravlik, Uspekhi Fizicheskikh Nauk 183, 287 - 306 (2013).

13. G. Mare, K. Dransfeld, Strong and Ultrastrong Magnetic Fields and Their Applications, ed. by F. Herlach, Springer Verlag, Berlin Heidelberg -New York - Tokyo (1985).

14. V.N.Samofalov, E.I Ilyashenko., A.Ramstad, L.Z. Lub yanuy, T.H. Johansen J. Optoelectr. Anv. Mat., 6, 911 (2004).

15. Patent US 7,492,550, (2009).

16. E.A. Nesbitt, J.H. Wernick, Rare Earth Permanent Magnets, Academic Press, New York London (1973).

17. E.C. Stoner, E.P. Wohlfart, Phil. Trans Roy. Soc., 240, 599 (1948).

18. I.E. Tamm, Fundamental Theory of Electricity, Nauka, Moscow (1966) [in Russian]. 